\title{
1 Keratinocyte desmoglein 1 regulates the epidermal microenvironment and tanning response
}

2

3 Christopher R. Arnette ${ }^{1}$, Jennifer L. Koetsier ${ }^{1}$, Joshua A. Broussard ${ }^{1,2}$, Pedram Gerami ${ }^{1,2,3,4}$, Jodi L.

4 Johnson ${ }^{1,2, *}$, and Kathleen J. Green ${ }^{1,2,4, *}$

5

$6 \quad{ }^{1}$ Department of Pathology, ${ }^{2}$ Department of Dermatology, ${ }^{3}$ Department of Pediatrics, and the ${ }^{4}$ Lurie

7 Comprehensive Cancer Center, Northwestern University, Feinberg School of Medicine, Chicago,

$8 \quad$ Illinois, 60611.

9

10 Running title: Dsg1 regulates melanocyte behavior

11

12 Key words: Desmosomal cadherin, Ultraviolet, Melanocyte, Cytokine

14 Abbreviations:

15 KC - Keratinocyte

16 MC - Melanocyte

17 Dsg1 - Desmoglein 1

18 UV - Ultraviolet light

19 E-cad - Epithelial cadherin

20 SAM - Severe dermatitis, multiple allergies, and metabolic wasting 
$21{ }^{*}$ Correspondence should be addressed to:

22 Jodi L. Johnson, Ph.D.

23 Department of Pathology Room 3-732. Northwestern University, Feinberg School of Medicine.

24303 E Chicago Ave., Chicago, IL 60611

25 Telephone: 312-503-5069; Fax: 312-503-8240

26 Email: jodi-johnson@northwestern.edu

27

28 Kathleen J. Green, Ph.D.

29 Department of Pathology W127, Room 3-735. Northwestern University, Feinberg School of

30 Medicine. 303 E Chicago Ave., Chicago, IL 60611

31 Telephone: 312-503-5300; Fax: 312-503-8240 or 312-503-8249

32 Email: kgreen@northwestern.edu 


\section{ABSTRACT}

35 Coordinated responses to environmental stimuli within the keratinocyte:melanocyte niche are poorly

36 understood. Desmoglein 1 (Dsg1), a keratinocyte-specific desmosomal cell-cell adhesion protein with

37 emerging signaling roles, is reduced by ultraviolet light radiation. Loss-of-function Dsg1 mutations

38 elevate keratinocyte cytokines in Severe dermatitis, multiple Allergies, and Metabolic wasting (SAM)

39 syndrome. We asked whether Dsg1 regulates keratinocyte:melanocyte paracrine communication to

40 induce the tanning response. Dsg1-silenced keratinocytes increased Pro-opiomelanocortin mRNA

41 and cytokine secretion. Melanocytes treated with conditioned media from Dsg1-silenced

42 keratinocytes exhibited increased Mitf and Trp1 mRNA, melanin secretion, and dendrite length.

43 Inhibiting the melanocyte pigment-associated melanocortin 1 receptor reduced pigment secretion in

44 response to Dsg1-deficient conditioned media. Melanocytes incorporated into Dsg1-deficient human

45 skin equivalents relocalized suprabasally, reminiscent of early melanoma pagetoid behavior. Dsg1

46 decreased in keratinocytes surrounding dysplastic nevi and early melanoma, but not benign nevi. We

47 posit Dsg1 controls keratinocyte:melanocyte communication through paracrine signaling, which goes

48 awry upon Dsg1 loss in melanoma development. 
57

58

59

60

61

62

63

64

65

66

67

68

69

70

71

72

73

74

75

76

77

78

79

80

81

\section{INTRODUCTION}

The epidermis is a multi-layered organ composed of several cell types that form a barrier against environmental, pathogenic, chemical, and physical assaults and against water loss. Keratinocytes (KCs), the major cell type of the epidermis, undergo a program of differentiation whereby they transit out of the proliferative basal cell layer and exit the cell cycle, giving rise to suprabasal layers (spinous, granular, and stratum corneum) (Jensen and Proksch, 2009; Proksch et al., 2008). Melanocytes (MCs), the pigment producing cells of the epidermis, reside within the basal layer of the skin. Through the extension of dendrites, MCs interact with KCs in a ratio of roughly 36 KCs: 1 MC, forming the KC:MC pigmentary unit (Nguyen and Fisher, 2018; Weiner et al., 2014). KCs and MCs directly associate through cadherin-based adhesive structures and indirectly communicate via paracrine signaling (Lee and Herlyn, 2007; Mescher et al., 2017; Serre et al., 2018). The release of secreted factors (cytokines, chemokines, and growth factors) from KCs and other resident skin cells results in modulation of MC proliferation, differentiation, signaling, pigment production and secretion, and dendricity (Yuan and Jin, 2018).

The KC:MC unit acts as a first line of defense to respond to environmental stimuli including ultraviolet (UV) light from the sun. Melanin transfer to KCs is a critical protective response that helps prevent UV-induced DNA damage and subsequent mutagenesis leading to cancer (Brenner and Hearing, 2008b; Yamaguchi et al., 2006). Activation of the MC transcription factor MITF in response to secretion of KC-derived factors (e.g. $\alpha-\mathrm{MSH}, \mathrm{KITL}$, ET1; Melanocyte stimulating hormone, Kit ligand, Endothelin 1) following UV exposure results in upregulation of melanin-producing enzymes (including TRP1; Tyrosinase related protein 1), and melanin production and secretion (Nguyen and Fisher, 2018; Serre et al., 2018). UV exposure also initiates signaling cascades in both KCs and MCs, resulting in secretion of cytokines and chemokines including interleukins (IL1, 3, 6, 8), interferon (IFN $\gamma$ ), granulocyte-colony stimulating factor (G-CSF), and tumor necrosis factor (TNF $\alpha$ ) (Schwarz and Luger, 1989; Terazawa and Imokawa, 2018; Yoshizumi et al., 2008), all of which control 
82 multicellular reactions such as the tanning response within the KC:MC unit. Lengthened dendrites in

83 response to UV light increase MC interactions with KCs to facilitate melanin transfer (Lopez et al.,

84 2015; Weiner et al., 2014). Further, secreted factors from KCs function to increase DNA damage

85 repair pathways in MCs, reducing the risk of oncogenic mutations (Kadekaro et al., 2010; Swope and

86 Abdel-Malek, 2016).

While UV light can modulate expression of cell-cell adhesion proteins (Dusek et al., 2006;

88 Jamal and Schneider, 2002; Johnson et al., 2014), the signaling roles of cadherin proteins have not

89 been fully explored. In particular, the UV-sensitive (Johnson et al., 2014), KC-specific desmosomal cadherin desmoglein 1 (Dsg1) has been linked to both cell adhesion and signaling as well as regulation of cytokines under homeostatic and pathologic conditions (Hammers and Stanley, 2013; Samuelov et al., 2013). Outside of its adhesive function, Dsg1 is an important regulator of cellular signaling, specifically in attenuating the MAPK/ERK pathway to promote KC differentiation (Getsios et al., 2009; Harmon et al., 2013). Activation of the MAPK/ERK pathway increases epidermal inflammation following UV exposure (El-Abaseri et al., 2013). Mutations in Dsg1 lead to Severe dermatitis, multiple Allergies, and Metabolic wasting (SAM) syndrome, accompanied by increased

97 pro-allergy cytokines (Samuelov et al., 2013). Also, several pathological conditions which result in 98 downregulation of Dsg1 at the cell surface can induce cytokine production, suggesting a role for Dsg1 99 in this process (Hammers and Stanley, 2013; Ishii et al., 2001; Polivka et al., 2018; Sherrill et al., 100 2014). Based on these previous studies, we questioned whether reduction of Dsg1, such as occurs 101 following UV exposure or other environmental stimuli, could mediate changes in paracrine 102 communication between KCs and MCs known to occur in response to these stimuli. microenvironment and MC behavior through regulation of cytokines, chemokines, and other secreted factors, thereby contributing to the epidermal tanning response. Using conditioned media from KCs 
107 signaling, pigment secretion, and dendricity. An increase in paracrine signaling from KCs resulted in

108 increased pigment secretion and altered dendrite length in MCs. We also utilized 3D human skin 109 equivalents containing both KCs and MCs to study the role of Dsg1 reduction in MC localization 110 within the epidermis, finding that suppression of Dsg1 resulted in mislocalization of MCs into the 111 suprabasal layers. This study elucidates a new role for Dsg1 in regulating KC:MC cell-cell 112 communication, and suggests a specific mechanism by which the epidermis senses and propagates 113 signals in response to environmental stimuli, which when perturbed can result in MC dysplasia.

\section{Suppression of KC Dsg1 increases cytokine production and secretion.}

We first sought to determine which cytokines were altered upon Dsg1 suppression in KCs.

118 KCs infected with retrovirus expressing non-target small hairpin RNA (NTshRNA), Dsg1-shRNA, 119 or Dsg1-shRNA reconstituted with silencing resistant Dsg1-Flag (Dsg1FL) were grown to 120 confluence, switched to high calcium medium to induce differentiation, and assessed by qRT-PCR 121 after 3 days. Cytokine transcripts including IL1 $\alpha$, IL1 $\beta$, IL6, IL8, and CXCL1 were significantly upregulated, IL2, IL4, and IL10 were significantly downregulated, and IL17, IL19, IL23, TNF $\alpha$ and

123 IFN $\gamma$ were highly variable or not significantly altered by suppression of Dsg1 (Figure 1A, additional 124 targets in Table 1). When a subset of these targets were tested, we found them to be returned to control 125 levels upon restoration of Dsg1FL (Figure 1A). Depletion of another desmosomal cadherin in KCs, 126 Dsg3, did not result in significant changes in tested cytokine mRNA levels (Figure 1B). conditioned days 3-5 (a 48 hour time period) after initiation of differentiation by growth in high 129 calcium medium was collected from KCs infected with NTshRNA or Dsg1shRNA. Dot-blot analysis 130 of 42 targets revealed increased secretion of GRO, CXCL1, IL6, and IL8 in the Dsg1shRNA 131 conditioned media compared to NTshRNA conditioned media (Figure 1C). KCs exposed to UV light 
132 have been reported to increase secretion of IL6 and IL8 among other cytokines/chemokines (Schwarz

133 and Luger, 1989; Yoshizumi et al., 2008). Since Dsg1 is downregulated in KCs following UV

134 exposure (Johnson et al., 2014) and its suppression results in increased cytokine/chemokine secretion,

135 we hypothesized that Dsg1 may play a role in coordinating the skin's paracrine response to 136 environmental insults.

137 Exposure to Dsg1-deficient KC conditioned media increases MC pigment secretion through classic

138 ligand-dependent mechanisms.

Since induction of pigment production and secretion in MCs is a crucial protective response in the epidermis that can be regulated by paracrine signaling (Serre et al., 2018; Yuan and Jin, 2018), we next tested MC pigment secretion following incubation with conditioned media from KCs with normal or suppressed Dsg1 expression. MCs were grown for 7 days in a 1:1 mixture of MC media and media conditioned by KCs days 3-5 after initiation of differentiation. Fresh 1:1 mixture was added to the culture every 2 days (none of the previously depleted media was removed). On day 7 , media was harvested and melanin secretion was analyzed by testing absorbance at $405 \mathrm{~nm}$ (Laskin et below the graph (Figure 2A), there was notable clonal variability in coloration of pigment secretion,

149 but all tested MC clones responded to incubation with Dsg1-deficient conditioned media by 150 increasing pigment secretion (Compiled data presented in Supplemental Figure 1).

151 To determine whether classical inducers of pigment production and secretion were induced in 152 KCs upon Dsg1 silencing, we tested for mRNA expression levels of Pro-opiomelanocortin (POMC 153 - precursor for the melanocortin 1 receptor [MC1R] ligand $\alpha-\mathrm{MSH}), \mathrm{KITL}$, and ET1. POMC was 154 increased upon Dsg1 suppression in KCs while KITL was not (Figure 2B). ET1 was highly variable 155 (data not shown). We tested induction of Mitf and Trp1 in MCs treated with conditioned media, 156 finding both to be elevated in MCs incubated with conditioned media deficient in Dsg1 (Figure 2C). 
157 When MCs were incubated with human beta defensin 3 (BD3) to inhibit MC1R (Swope et al., 2012;

158 Wolf Horrell et al., 2016), pigment induction was inhibited downstream of Dsg1-deficient 159 conditioned media (Figure 2D). Together these results indicate that reduction of Dsg1 in KCs results 160 in induction of the classical pigment production pathways in MCs dependent upon paracrine signaling 161 from KCs.

Dsg1-deficient KCs change MC dendricity, partially dependent upon cytokine/chemokine signaling. deficient KC conditioned media resulted in increased dendrite length as compared to those in NTshRNA or Dsg1FL-conditioned media (Figure 3A), similar to the response seen following UV exposure and an important determinant of the number of KCs that a MC can interact with in normal

171 skin (Lopez et al., 2015; Weiner et al., 2014). However, 7 days of exposure to Dsg1-deficient KC 172 conditioned media resulted in shortening of MC dendrites (Figure 3A lower). the observed changes in MC dendricity. We inhibited the CXCL1/IL8 receptor (CXCR2) using

175 Reparixin, which inhibited the increase in MC dendrite length at the 12 hour time point upon exposure to Dsg1-deficient conditioned media (Figure 3B). We also queried whether recombinant IL6, IL8, or

177 a combination of the 2 added into base MC media for either 12 hours or 7 days were sufficient to 178 impact dendrite length. While neither individual cytokine was sufficient to alter dendrite length at the 17912 hour time point, MC dendrites significantly lengthened in the presence of IL6, but not IL8 after 180 incubation for 7 days (Figure 3C). This observation is consistent with the possibility that individual 181 cytokines contribute to MC dendrite length changes, but are not sufficient to recapitulate the time 
182

183

184

185

course and/or signal propagation stimulated by the complete Dsg1-deficient conditioned media. This may indicate that multiple paracrine factors are required to elicit the MC dendrite changes observed upon addition of Dsg1-deficient conditioned media. Together these data indicate that cytokine/chemokine signaling is at least partially responsible for altering MC dendrite length downstream of Dsg1 suppression.

\section{MCs are mislocalized within the $3 D$ skin structure when KC Dsg1 is reduced.}

The 3D architecture of the skin is an important contributor to how signals are propagated throughout the tissue and represents a complex integration of multiple signaling and adhesive cues that differ from when cells are grown in 2D culture (Li et al., 2011). We assessed whether reduction of KC Dsg1 in 3D organotypic cultures containing both KCs and MCs would result in changes in MC behavior. Either NTshRNA or Dsg1shRNA-infected KCs were seeded with MCs at a physiological ratio of 36:1, grown, and lifted to the air-liquid interface to allow stratification for six days. MCs were mis-localized from the basal to suprabasal layers in KC Dsg1-deficient organotypic cultures (Figure 4A). Imaging of organotypic cultures (shCon, shDsg1; representative immunoblot Figure 4B) prepared using the whole mount method revealed the extent to which melanocytes moved into the upper epidermal layers throughout the entire organotypic culture rather than in one plane of section (Figure 4C).

Dsg1 levels are significantly reduced at cell-cell borders in the KCs surrounding human dysplastic nevi and melanoma.

The observed changes in MC dendrite length, localization, and cytokine profiles when exposed to conditioned media from KCs with suppressed Dsg1 are reminiscent of MCs that have undergone transformation (Colebatch and Scolyer, 2018; Dhawan and Richmond, 2002). Increased MITF levels have also been associated with MC transformation and melanoma progression (Hartman and Czyz, 2015). We therefore examined Dsg1 levels in KCs surrounding benign and dysplastic 
206 pigmented nevi and in early stage melanomas to determine if Dsg1 suppression was a phenomenon

207 observable in the progression of human disease. To assess the global levels of Dsg1 at KC cell-cell

208 borders in normal human skin and melanoma samples, tissue sections were stained for Dsg1 and the

209 intensity measured. Control skin and benign MC nevi exhibited similar levels of Dsg1 at cell-cell

210 borders (Figure 5A, 5B upper). However, Dsg1 was found to be significantly decreased in matched

211 dysplastic nevi and melanoma samples from the same individuals compared to normal skin. Epithelial

212 cadherin, (Ecad) was not significantly reduced in the same KC populations, indicating that reduction

213 in Dsg1 precedes the previously reported reduction in Ecad in human melanoma (Figure 5A, 5B

214 lower) (Lee and Herlyn, 2007). Together our data are consistent with the idea that decreased KC Dsg1

215 levels as occurs following UV exposure (Johnson et al., 2014) alter the microenvironment within the

216 KC:MC unit to propagate the tanning response. We posit that longer-term Dsg1 suppression, as

217 observed surrounding human dysplastic and melanoma lesions may contribute to melanocyte

218 transformation.

\section{DISCUSSION}

221 Our data support a model where Dsg1, important in epidermal cell-cell adhesion, also

222 functions as a rheostat to sense and respond to environmental insults; reduced Dsg1 expression such

223 as occurs following UV light exposure (Johnson et al., 2014) increases paracrine signaling from KCs

224 to help initiate the MC tanning response (Figure 6). The regulation of MCs via secretion of paracrine

225 factors by KCs is a well-established phenomenon (Serre et al., 2018; Yuan and Jin, 2018). Our study

226 places Dsg1 as a key regulator of the microenvironment within the KC:MC unit.

Protection against damage from environmental exposure, including UV light from the sun is

228 one of the key barrier functions of the epidermis. The classic KC:MC tanning response protects

229 against mutagenesis (Brenner and Hearing, 2008a; Kadekaro et al., 2010; Swope and Abdel-Malek, 
2016; Yamaguchi et al., 2006) and involves KC increase of POMC, production and secretion of aMSH as well as other secreted factors, and subsequent activation of the MC receptor MC1R,

232 downstream activation of MITF, and pigment production through TRP1 and TRP2 (Serre et al., 233 2018). All human skin, regardless of skin type or melanin type and quantity (Wakamatsu et al., 2006)

234 undergoes reaction to UV exposure including increased pigmentation (Alaluf et al., 2001). A recent 235 study suggested that MCs from darkly pigmented individuals more effectively increased 236 melanogenesis after UVB exposure in the presence of KC conditioned media compared to MCs from 237 lightly pigmented individuals, suggesting an enhanced photoprotective mechanism in darker skin types (Lopez et al., 2015). Though a full study of MC clones from light and darkly pigmented individuals was not undertaken here, we show that reduction in KC Dsg1, such as occurs following UVB light exposure (Johnson et al., 2014) increases pigment secretion in MCs from donors with multiple skin types.

MC dendrites are crucial for mediating the tanning response following UV exposure since they are the vehicle through which melanosomes are transferred to multiple KCs (Gilchrest et al., 1996). We showed that factors present in Dsg1-deficient conditioned media can signal to MCs to alter 245 dendrite length. UV exposed KCs increase secretion of IL6 and IL8, similar to our findings of 246 cytokine secretion downstream of Dsg1 suppression (Schwarz and Luger, 1989; Yoshizumi et al., 247 2008). We showed that IL6 and the IL8/CXCL1 receptor (CXCR2) both impact melanocyte 248 dendricity downstream of Dsg1. However, addition of IL6 or inhibition of CXCR2 were both 249 insufficient to recapitulate the precise effects and timeline of complete Dsg1-deficient KC media. 250 Therefore, further studies are needed to elucidate the roles of these and other cytokines and 251 chemokines in regulating the dendrite morphology of MCs downstream of Dsg1. 
repeated cycles of acute UV exposure could reduce Dsg1 expression in KCs for prolonged amounts of time, leading to alternations in other KC:MC adhesive molecules (Mescher et al., 2017) or an altered microenvironment within the KC:MC unit to promote MC dysplasia.

Nests of KCs surrounding human dysplastic and early stage melanoma lesions exhibited decreased levels of Dsg1 at cell-cell borders which preceded the previously reported loss of Ecad during melanoma progression (Lee and Herlyn, 2007). We show here that normal KCs lacking Dsg1

261 increase secretion of IL6, IL8, and CXCL1, all of which have been implicated in melanoma 262 development and maintenance (Jobe et al., 2016; Payne and Cornelius, 2002). While our study implicates Dsg1 loss in regulation of the protective epidermal tanning response, the possibility exists that repeated reduction in KC Dsg1, such as through repeated acute UV exposure or through an increased epidermal oxidative environment (Wittgen and van Kempen, 2007), ultimately leads to a pro-tumorigenic microenvironment in vivo. Transformed MCs themselves could secrete factors resulting in more sustained loss of KC Dsg1. Cytokine secretion and altered inflammatory microenvironment contributes to the growth, invasion, and angiogenesis of transformed MCs and melanoma cells (Richmond et al., 2009). In fact, CXCL1 was identified as one of the most highly modified markers allowing discrimination between common melanocytic and dysplastic nevi (Mitsui

271 et al., 2016). Identifying KC Dsg1 as a regulator of MC pigment secretion, dendricity, and position 272 within epidermis places Dsg1 at a nexus of regulating normal homeostasis within the KC:MC unit, 273 and suggests how this can go awry in nevi to melanoma progression as MCs themselves begin to alter 274 the microenvironment, questions which have remained open in the field for decades and remain open 275 still (Colebatch and Scolyer, 2018; Haass and Herlyn, 2005). 
et al., 2018). Other desmogleins are being regulated through use of glucocorticoids, rapamycin, and

281 Stat3 inhibition in treatment of pemphigus (Mao et al., 2017). Therefore, pharmacological modulation

282 of Dsg1 expression is possible with potential ramifications for clinically managing pigmentation or

283 reducing melanomagenesis from dysplastic nevi.

In conclusion, this study demonstrates a new role for KC Dsg1 in regulating paracrine crosstalk within the epidermal KC:MC unit. Reduction in Dsg1 results in KCs increasing both liganddependent and cytokine/chemokine-dependent signaling to MCs to stimulate MC pigment production and secretion, and dendricity. Within the 3D epidermis, the presence of KC Dsg1 helps properly position MCs in the basal layer; Dsg1 reduction results in suprabasal mislocalization of MCs, which may be involved in the trajectory from benign to dysplastic nevi formation, since KC Dsg1 loss occurs surrounding human dysplastic nevi and melanoma lesions.

MATERIALS AND METHODS

\section{Cell culture and retroviral transduction}

KCs and MCs were isolated from neonatal foreskin provided by the Northwestern University Skin

Disease Research Center (NUSDRC) as described in (Halbert et al., 1992). KCs were propagated in M154 medium supplemented with human KC growth supplement (Life Technologies, Grand Island,

$297 \mathrm{NY}$ ), 1,000 x gentamycin/amphotericin B solution (Life Technologies, and 0.07mM CaCl 2 (low 298 calcium). Confluent $\mathrm{KC}$ monolayers were induced to differentiate by the addition of $1.2 \mathrm{mM} \mathrm{CaCl}_{2}$

299 (high calcium) in M154 in the presence of human KC growth supplement. KCs were transduced with 300 retroviral supernatants produced from Phoenix cells (provided by G. Nolan, Stanford University, 301 Stanford, CA) as previously described (Getsios et al., 2004; Simpson et al., 2010b). MCs were 302 cultured in OptiMEM (Life Technologies, Carlsbad, CA) containing 1\% penicillin/streptomycin 303 (Corning, Corning, NY), 5\% fetal bovine serum (Sigma-Aldrich, St. Louis, MO), 10 ng/ml bFGF 
304 (ConnStem Inc., Cheshire, CT), 1 ng/ml heparin (Sigma-Aldrich), $0.1 \mathrm{mM} \mathrm{N}$ N $^{6}$ 2'-O-

305 dibutyryladenosine 3:5-cyclic monophosphate (dbcAMP; Sigma-Aldrich), and 0.1 mM 3-isobutyl-1-

306 methyl xanthine (IBMX; Sigma-Aldrich).

307 Organotypic skin cultures and whole mount samples

308 Organotypic cultures were grown as described previously (Arnette et al., 2016; Getsios et al., 2009)

309 with the addition of the following steps: the collagen plug was coated with extracellular matrix

310 using 804G supernatant (Langhofer et al., 1993). MCs were seeded onto the collagen plug

311 overnight. KCs were then seeded at a physiologic ratio of approximately $36 \mathrm{KCs}: 1 \mathrm{MC}$.

312 Organotypic cultures fixed in 10\% neutral buffered formalin were embedded in paraffin blocks and

313 cut into $4 \mu \mathrm{m}$ sections. For indirect immunofluorescence microscopy, slides were baked at $60^{\circ} \mathrm{C}$,

314 de-paraffinized by xylenes, dehydrated with ethanol, rehydrated in PBS and permeablized by 0.5\%

315 Triton X-100 in PBS. Antigen retrieval was performed by incubation in $0.01 \mathrm{M}$ Citrate buffer (pH

316 6.0) at $95^{\circ} \mathrm{C}$ for 15 minutes. Sections were blocked in $1 \%$ BSA/2\% Normal Goat Serum/PBS for 30

317 minutes at $37^{\circ} \mathrm{C}$. Primary antibody incubation was carried out overnight at $4^{\circ} \mathrm{C}$ in blocking buffer

318 followed by washing in PBS. Secondary antibody incubation was carried out at $37^{\circ} \mathrm{C}$ for 45 minutes

319 followed by washing in PBS. Sections were stained with 4',6-Diamidino-2-phenylindole (DAPI -

320 Sigma-Aldrich) at a final concentration of $5 \mathrm{ng} / \mu \mathrm{l}$ at room temperature for 2 minutes followed by

321 washing in PBS and water. Cover slips were mounted on the sections with ProLong Gold Antifade

322 Reagent (Invitrogen, Life Technologies).

323 For whole mount imaging: Six days after lifting to the air-liquid interface, the epidermal equivalent

324 layer was removed from the collagen plug and fixed in 4\% paraformaldehyde in PBS for 15 min on

325 ice. Samples were then washed three times in PBS for 5 min each at room temperature.

326 Subsequently, samples were incubated in blocking buffer (1\% Triton-X 100 with 5\% goat serum in

327 PBS) for 1 hr at $37^{\circ} \mathrm{C}$ followed by incubation overnight at $37^{\circ} \mathrm{C}$ with S100 (ab52642, anti-S100

328 beta; Abcam, Cambridge, UK) diluted at 1:100 in blocking buffer. Samples were washed 3 times

329 for 10 min each with PBS at room temperature and then incubated overnight at $37^{\circ} \mathrm{C}$ with Alexa 

onto glass slides with Prolong Gold Antifade Reagent.

\section{Preparation of conditioned media from KCs}

334 KCs infected at 20\% confluence with LZRS-miR Dsg1, LZRS-NTshRNA, LZRS-Flag Dsg1, and

$20^{\circ} \mathrm{C}$ in aliquots until needed for an experiment.

\section{DNA constructs}

LZRS-miR Dsg1 (Dsg1shRNA), LZRS-Flag Dsg1, and LZRS-Dsg1 $\Delta 381$ were generated as

\section{Antibodies and reagents}

348 The mouse monoclonal antibodies used were as follows: P124 (anti-Dsg1 extracellular domain;

349 Progen, Heidelberg, Germany), 27B2 (anti-Dsg1 cytodomain; Life Technologies), and HMB45

350 (Melanoma gp100 antibody; Thermo Fisher Scientific). The rabbit monoclonal antibody EP1576Y

351 (ab52642, anti-S100 beta; Abcam, Cambridge, UK) was used. Rabbit polyclonal antibodies used

352 were: HECD1 (anti-E-cadherin; Takara, Kyoto, Japan), Flag (Cell Signaling Technologies), Anti-

353 MelanA (ab15468), and GAPDH (G9545, glyceraldehyde-3-phosphate dehydrogenase; Sigma-

354 Aldrich). Secondary antibodies for immunoblotting were goat anti-mouse and goat anti-rabbit 
355 peroxidase (Rockland; KPL, Gaithersburg, MD). Secondary antibodies for immunofluorescence were

356 goat anti-mouse and goat-anti-rabbit linked to fluorophores of 488 and $568 \mathrm{~nm}$ (Alexa Fluor; Life

357 Technologies). DAPI was used to stain nuclei.

358 Western blot analysis of proteins

359 Whole cell lysates were collected from confluent monolayers in urea-SDS buffer (8 M urea/1\%

360 SDS/60 mM Tris (pH 6.8)/5\% ß-mercaptoethanol/10\% glycerol) and sonicated. Samples separated

361 by SDS-PAGE were transferred to nitrocellulose, blocked in 5\% milk/PBS, and incubated with

362 primary antibodies in milk for 1 hour at room temperature or overnight at $4^{\circ} \mathrm{C}$. After a series of PBS

363 washes, secondary antibodies diluted in milk were added to blots. Protein bands were visualized

364 using exposure to X-ray film. Densitometric analyses were performed of scanned immunoblots

365 using ImageJ software.

366 Dot Blot Analysis of Cytokines

367 Conditioned media from KCs (day 3-5 conditioned media) was concentrated 5x by centrifugation

368 through a Centricon Plus-70 Centrifugal Filter with a cutoff of 3 kDa (Millipore Sigma, St Louis,

369 MO) according to the manufacturer's instructions. Protein concentration was normalized and a 1

$370 \mathrm{~mL}$ sample was incubated according to the manufacturer's instructions on a Human Cytokine C3

371 Array (Raybiotech, Norcross, GA) capable of detecting 42 human cytokines and chemokines.

372 Quantitative real-time PCR

373 RNA was isolated from KCs and MCs using the RNeasy Mini Kit (Qiagen, Valencia, CA), according

374 to the manufacturer's instructions. MCs were grown in monoculture prior to being incubated

375 overnight or for 7 days with either MC media alone or with a 1:1 mixture of MC media and

376 conditioned media. Total RNA concentrations were equalized between samples and cDNA was

377 prepared using the Superscript III First Strand Synthesis Kit (Life Technologies). Quantitative PCR

378 was performed using SYBR Green PCR master mix (Life Technologies) and gene-specific primers 
379 (Table 2) in a StepOnePlus instrument. Calculations for relative mRNA levels were performed using

380 the $\Delta \Delta \mathrm{Ct}$ method, normalized to GAPDH.

$381 \quad$ Microscope imaging

382 Wide-field images were acquired on an Upright Leica microscope (model DMR) fitted with an Orca-

383100 digital camera (model C4742-95; Hamamatsu Photonics) and a 40x 1.0 numerical aperture (NA)

384 oil Plan Fluotar objective. Apotome images were acquired using an epifluorescence microscope

385 system (AxioVision Z1; Carl Zeiss, Thornwood, NY) fitted with an Apotome slide module, AxioCam

386 MRm digital camera, and a 40x 0.5 EC Plan-Neofluar or 100x 1.4 NA oil Plan-Apochromat objective

387 (Carl Zeiss). For each experiment, images were acquired using the same imaging conditions.

388 Confocal z-stacks (z-step size of $0.5 \mu \mathrm{m}$ ) of whole mount samples were acquired using a

389 Nikon A1R confocal laser microscope equipped with GaAsP detectors and a 60× Plan-Apochromat

390 objective lambda with a NA of 1.4 and run by NIS Elements software (Nikon). NIS Elements

391 (version 5.02) was used to generate 3D reconstructions of z-stacks using the Volume Viewer tool

392 with z-depth coding blending and the Rainbow contrast look up table and to determine the $\mathrm{z}$

393 position of the melanocyte cell body centroid.

394 Fluorescence intensity of cell-cell borders

395 For each image, background intensity was defined as the average of three mean pixel intensity 396 measurements from an image without fluorescence. Fluorescence pixel intensity at random KC 397 borders surrounding a MC or a cluster of MCs was determined by measuring the mean pixel intensity 398 at a defined border and dividing by the border length.

399 Quantification of dendrite length

400 MCs were grown to $75 \%$ confluence in MC media and subsequently switched to a 1:1 ratio of KC

401 conditioned media or continued to be grown in MC media alone. Cells were incubated overnight 402 (12hrs) or for 7 days before being fixed in 4\% paraformaldehyde (Sigma-Aldrich) in PBS for 10 403 minutes. Wide-field images were used to visualize MC dendrites following incubation. Dendrite 404 parameters were quantified with Fiji software (NIH). Dendrites were defined as extensions 
al., 2007) (Cayman Chemical, Ann Arbor, MI) was added to media at a final concentration of $5 \mu \mathrm{g} / \mathrm{ml}$ media. Recombinant IL6 and IL8 (Abcam) were added to media at final concentrations of $0.2 \mathrm{ng} / \mathrm{ml}$ and $25 \mathrm{ng} / \mathrm{ml}$, respectively.

\section{Quantification of melanin secretion}

411 MCs were grown to confluence in MC media and subsequently switched to a 1:1 ratio of KC

412 conditioned media or media alone with fresh media added to existing media every 2 days up to 7

413 days. After 7 days, media was collected and subjected to centrifugation at 11,000 rpm for 1 minute.

414 The resulting melanosome pellet was re-suspended in $200 \mu \mathrm{l} \mathrm{PBS}$ and transferred to the appropriate

415 well of a 96-well plate (BD Biosciences). The absorbance at $405 \mathrm{~nm}$ of each well was measured with

416 an ELx800 microplate reader (Bio-Tek Instruments, Inc). Absorbance readings were plotted after

417 normalizing to a media only control for each condition in 3 independent experiments. When

418 applicable, 100 nM Recombinant Human Beta Defensin 3 (Sigma Aldrich, Saint Louis, MO) was

419 used to inhibit MC1R (Swope et al., 2012; Wolf Horrell et al., 2016).

\section{Sample size and statistical analysis}

421 All experiments were performed independently $\geq 3$ times (i.e. biological replicates performed on

422 different days, not technical replicates performed in parallel at the same time). For each

423 independent/biological replicate, multiple experimental/control arms were processed and analyzed

424 in parallel. Representative experiments are displayed throughout the figures. All graphs are

425 displayed as mean \pm standard error of the mean (SEM). Power analyses to determine sample sizes

426 were determined in consultation with the Northwestern University Quantitative Data Sciences Core.

427 Two group comparisons were performed using two-tailed, two-sample equal variance Student's t

428 test. For comparisons of more than two groups, One-way ANOVA on ranks (Kruskal-Wallis) was

429 used, followed by Tukey or Dunn’s multiple comparisons test. All analysis was performed using 
430 GraphPad Prism version 7.0 for Mac (GraphPad Software, La Jolla, CA, USA). Statistical

431 significance was defined as, ${ }^{*} \mathrm{p}<0.03,{ }^{* *} \mathrm{p}<0.002,{ }^{* * *} \mathrm{p}<0.0002,{ }^{* * * *} \mathrm{p}<0.0001$.

434 Acknowledgements: This work was supported by NIH/NIAMS R01 AR041836 and NIH/NCI R01

435 CA122151 to KJG and by the Liz and Eric Lefkofsky Family Foundation Innovation Research

436 Award to KJG and JLJ. Additional support was provided by the JL Mayberry endowment to KJG.

437 CRA was supported through a NIH/NCI Ruth L. Kirschstein Training Grant through Northwestern

438 University’s Robert H. Lurie Comprehensive Cancer Center (T32 CA070085-14) “Signal

439 Transduction in Cancer” and also through a NIH/NCI Ruth L. Kirschstein National Research

440 Service Award 1F32CA210498-01. We thank Dr. Zalfa Abdel-Malek (University of Cincinnati) for

441 critical reading of the manuscript. We acknowledge support and materials from the Northwestern

442 University Skin Disease Research Center supported by 5P30AR057216. Imaging work was

443 performed at the Northwestern University Center for Advanced Microscopy generously supported

444 by NCI CCSG P30 CA060553 awarded to the Robert H Lurie Comprehensive Cancer Center.

446 Conflict of Interest: The authors declare no conflicts of interest. 


\section{REFERENCES:}

448 Alaluf S, Heath A, Carter N, Atkins D, Mahalingam H, Barrett K, et al. Variation in melanin

449 content and composition in type V and VI photoexposed and photoprotected human skin: the

450 dominant role of DHI. Pigment Cell Res 2001;14:337-47.

451

452 Arnette C, Koetsier JL, Hoover P, Getsios S, Green KJ. In Vitro Model of the Epidermis:

453 Connecting Protein Function to 3D Structure. Methods Enzymol 2016;569:287-308.

454

455 Brenner M, Hearing VJ. Modifying skin pigmentation - approaches through intrinsic biochemistry

456 and exogenous agents. Drug Discov Today Dis Mech 2008a;5:e189-e99.

457

458 Brenner M, Hearing VJ. The protective role of melanin against UV damage in human skin.

459 Photochem Photobiol 2008b;84:539-49.

460

461 Colebatch AJ, Scolyer RA. Trajectories of premalignancy during the journey from melanocyte to

462 melanoma. Pathology 2018;50:16-23.

463

464 Dhawan P, Richmond A. Role of CXCL1 in tumorigenesis of melanoma. J Leukoc Biol 2002;72:9-

46518. 
467 Dusek RL, Getsios S, Chen F, Park JK, Amargo EV, Cryns VL, et al. The differentiation-dependent

468 desmosomal cadherin desmoglein 1 is a novel caspase-3 target that regulates apoptosis in

469 keratinocytes. J Biol Chem 2006;281:3614-24.

471 El-Abaseri TB, Hammiller B, Repertinger SK, Hansen LA. The epidermal growth factor receptor

472 increases cytokine production and cutaneous inflammation in response to ultraviolet irradiation.

473 ISRN Dermatol 2013;2013:848705.

475 Fournier JF, Bhurruth-Alcor Y, Musicki B, Aubert J, Aurelly M, Bouix-Peter C, et al. Squaramides

476 as novel class I and IIB histone deacetylase inhibitors for topical treatment of cutaneous t-cell

477 lymphoma. Bioorg Med Chem Lett 2018;28:2985-92.

478

479 Getsios S, Amargo EV, Dusek RL, Ishii K, Sheu L, Godsel LM, et al. Coordinated expression of

480 desmoglein 1 and desmocollin 1 regulates intercellular adhesion. Differentiation 2004;72:419-33.

481

482 Getsios S, Simpson CL, Kojima S, Harmon R, Sheu LJ, Dusek RL, et al. Desmoglein 1-dependent

483 suppression of EGFR signaling promotes epidermal differentiation and morphogenesis. J Cell Biol $484 \quad 2009 ; 185: 1243-58$.

486 Gilchrest BA, Park HY, Eller MS, Yaar M. Mechanisms of ultraviolet light-induced pigmentation.

487 Photochem Photobiol 1996;63:1-10. 
489 Haass NK, Herlyn M. Normal human melanocyte homeostasis as a paradigm for understanding

490 melanoma. J Investig Dermatol Symp Proc 2005;10:153-63.

491

492 Halbert CL, Demers GW, Galloway DA. The E6 and E7 genes of human papillomavirus type 6

493 have weak immortalizing activity in human epithelial cells. J Virol 1992;66:2125-34.

494

495 Hammers CM, Stanley JR. Desmoglein-1, differentiation, and disease. J Clin Invest 2013;123:1419-

49622.

497

498 Harmon RM, Simpson CL, Johnson JL, Koetsier JL, Dubash AD, Najor NA, et al. Desmoglein-

499 1/Erbin interaction suppresses ERK activation to support epidermal differentiation. J Clin Invest

$500 \quad$ 2013;123:1556-70.

501

502 Hartman ML, Czyz M. MITF in melanoma: mechanisms behind its expression and activity. Cell

503 Mol Life Sci 2015;72:1249-60.

504

505 Ishii K, Norvell SM, Bannon LJ, Amargo EV, Pascoe LT, Green KJ. Assembly of desmosomal

506 cadherins into desmosomes is isoform dependent. J Invest Dermatol 2001;117:26-35. 
508 Jamal S, Schneider RJ. UV-induction of keratinocyte endothelin-1 downregulates E-cadherin in

509 melanocytes and melanoma cells. J Clin Invest 2002;110:443-52.

511 Jensen JM, Proksch E. The skin's barrier. G Ital Dermatol Venereol 2009;144:689-700.

513 Jobe NP, Rosel D, Dvorankova B, Kodet O, Lacina L, Mateu R, et al. Simultaneous blocking of IL-

$514 \quad 6$ and IL-8 is sufficient to fully inhibit CAF-induced human melanoma cell invasiveness. Histochem

515 Cell Biol 2016;146:205-17.

517 Johnson JL, Koetsier JL, Sirico A, Agidi AT, Antonini D, Missero C, et al. The desmosomal protein

518 desmoglein 1 aids recovery of epidermal differentiation after acute UV light exposure. J Invest

519 Dermatol 2014;134:2154-62.

521 Kadekaro AL, Leachman S, Kavanagh RJ, Swope V, Cassidy P, Supp D, et al. Melanocortin 1

522 receptor genotype: an important determinant of the damage response of melanocytes to ultraviolet

523 radiation. FASEB J 2010;24:3850-60.

52764. 
529 Laskin JD, Piccinini L, Engelhardt DL, Weinstein IB. Control of melanin synthesis and secretion by

530 B16/C3 melanoma cells. J Cell Physiol 1982;113:481-6.

531

532 Lee JT, Herlyn M. Microenvironmental influences in melanoma progression. J Cell Biochem

$533 \quad 2007 ; 101: 862-72$.

534

535 Li L, Fukunaga-Kalabis M, Herlyn M. The three-dimensional human skin reconstruct model: a tool

536 to study normal skin and melanoma progression. J Vis Exp 2011;54:e2937.

537

538 Lopez S, Alonso S, Garcia de Galdeano A, Smith-Zubiaga I. Melanocytes from dark and light skin

539 respond differently after ultraviolet B irradiation: effect of keratinocyte-conditioned medium.

$540 \quad$ Photodermatol Photoimmunol Photomed 2015;31:149-58.

541

542 Mao X, Cho MJT, Ellebrecht CT, Mukherjee EM, Payne AS. Stat3 regulates desmoglein 3

543 transcription in epithelial keratinocytes. JCI Insight 2017;2:e92253.

544

545 Mescher M, Jeong P, Knapp SK, Rubsam M, Saynisch M, Kranen M, et al. The epidermal polarity

546 protein Par3 is a non-cell autonomous suppressor of malignant melanoma. J Exp Med

$547 \quad 2017 ; 214: 339-58$. 
549 Mitsui H, Kiecker F, Shemer A, Cannizzaro MV, Wang CQ, Gulati N, et al. Discrimination of

550 Dysplastic Nevi from Common Melanocytic Nevi by Cellular and Molecular Criteria. J Invest

551 Dermatol 2016;136:2030-40.

552

553 Moriconi A, Cesta MC, Cervellera MN, Aramini A, Coniglio S, Colagioia S, et al. Design of

554 noncompetitive interleukin-8 inhibitors acting on CXCR1 and CXCR2. J Med Chem 2007;50:3984-

5554002.

556

557 Nguyen NT, Fisher DE. MITF and UV responses in skin: From pigmentation to addiction. Pigment

558 Cell Melanoma Res 2018; doi:10.1111/pcmr.12726.

559

560 Payne AS, Cornelius LA. The role of chemokines in melanoma tumor growth and metastasis. J

561 Invest Dermatol 2002;118:915-22.

562

563 Polivka L, Hadj-Rabia S, Bal E, Leclerc-Mercier S, Madrange M, Hamel Y, et al. Epithelial barrier

564 dysfunction in desmoglein-1 deficiency. J Allergy Clin Immunol 2018;142:702-6 e7.

565

566 Proksch E, Brandner JM, Jensen JM. The skin: an indispensable barrier. Exp Dermatol

$567 \quad 2008 ; 17: 1063-72$. 
569 Richmond A, Yang J, Su Y. The good and the bad of chemokines/chemokine receptors in

570 melanoma. Pigment Cell Melanoma Res 2009;22:175-86.

571

572 Samuelov L, Sarig O, Harmon RM, Rapaport D, Ishida-Yamamoto A, Isakov O, et al. Desmoglein

5731 deficiency results in severe dermatitis, multiple allergies and metabolic wasting. Nat Genet

$574 \quad 2013 ; 45: 1244-8$.

575

576 Schwarz T, Luger TA. Effect of UV irradiation on epidermal cell cytokine production. J Photochem

577 Photobiol B 1989;4:1-13.

578

579 Serre C, Busuttil V, Botto JM. Intrinsic and extrinsic regulation of human skin melanogenesis and 580 pigmentation. Int J Cosmet Sci 2018; doi:10.1111/ics.12466.

582 Sherrill JD, Kc K, Wu D, Djukic Z, Caldwell JM, Stucke EM, et al. Desmoglein-1 regulates

583 esophageal epithelial barrier function and immune responses in eosinophilic esophagitis. Mucosal

584 Immunol 2014;7:718-29.

586 Simpson CL, Kojima S, Cooper-Whitehair V, Getsios S, Green KJ. Plakoglobin rescues adhesive

587 defects induced by ectodomain truncation of the desmosomal cadherin desmoglein 1: implications

588 for exfoliative toxin-mediated skin blistering. Am J Pathol 2010a;177:2921-37. 
590 Simpson CL, Kojima S, Getsios S. RNA interference in keratinocytes and an organotypic model of

591 human epidermis. Methods Mol Biol 2010b;585:127-46.

592

593 Swope VB, Abdel-Malek ZA. Significance of the Melanocortin 1 and Endothelin B Receptors in

594 Melanocyte Homeostasis and Prevention of Sun-Induced Genotoxicity. Front Genet 2016;7:146.

595

596 Swope VB, Jameson JA, McFarland KL, Supp DM, Miller WE, McGraw DW, et al. Defining

597 MC1R regulation in human melanocytes by its agonist alpha-melanocortin and antagonists agouti

598 signaling protein and beta-defensin 3. J Invest Dermatol 2012;132:2255-62.

599

600 Terazawa S, Imokawa G. Signaling Cascades Activated by UVB in Human Melanocytes Lead to

601 the Increased Expression of Melanocyte Receptors, Endothelin B Receptor and c-KIT. Photochem

602 Photobiol 2018;94:421-31.

603

604 Wakamatsu K, Kavanagh R, Kadekaro AL, Terzieva S, Sturm RA, Leachman S, et al. Diversity of

605 pigmentation in cultured human melanocytes is due to differences in the type as well as quantity of 606 melanin. Pigment Cell Res 2006;19:154-62.

607

608 Weiner L, Fu W, Chirico WJ, Brissette JL. Skin as a living coloring book: how epithelial cells

609 create patterns of pigmentation. Pigment Cell Melanoma Res 2014;27:1014-31. 
611 Wittgen HG, van Kempen LC. Reactive oxygen species in melanoma and its therapeutic

612 implications. Melanoma Res 2007;17:400-9.

613

614 Wolf Horrell EM, Boulanger MC, D'Orazio JA. Melanocortin 1 Receptor: Structure, Function, and

615 Regulation. Front Genet 2016;7:95.

616

617 Yamaguchi Y, Takahashi K, Zmudzka BZ, Kornhauser A, Miller SA, Tadokoro T, et al. Human

618 skin responses to UV radiation: pigment in the upper epidermis protects against DNA damage in the

619 lower epidermis and facilitates apoptosis. FASEB J 2006;20:1486-8.

620

621 Yoshizumi M, Nakamura T, Kato M, Ishioka T, Kozawa K, Wakamatsu K, et al. Release of

622 cytokines/chemokines and cell death in UVB-irradiated human keratinocytes, HaCaT. Cell Biol Int

$623 \quad 2008 ; 32: 1405-11$.

624

625 Yuan XH, Jin ZH. Paracrine regulation of melanogenesis. Br J Dermatol 2018;178:632-9.

626

627 
A

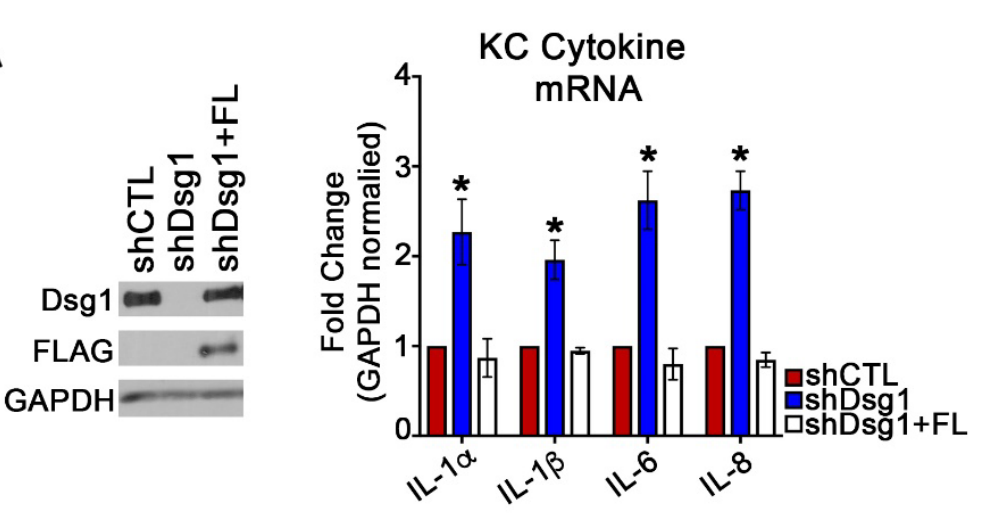

B


C
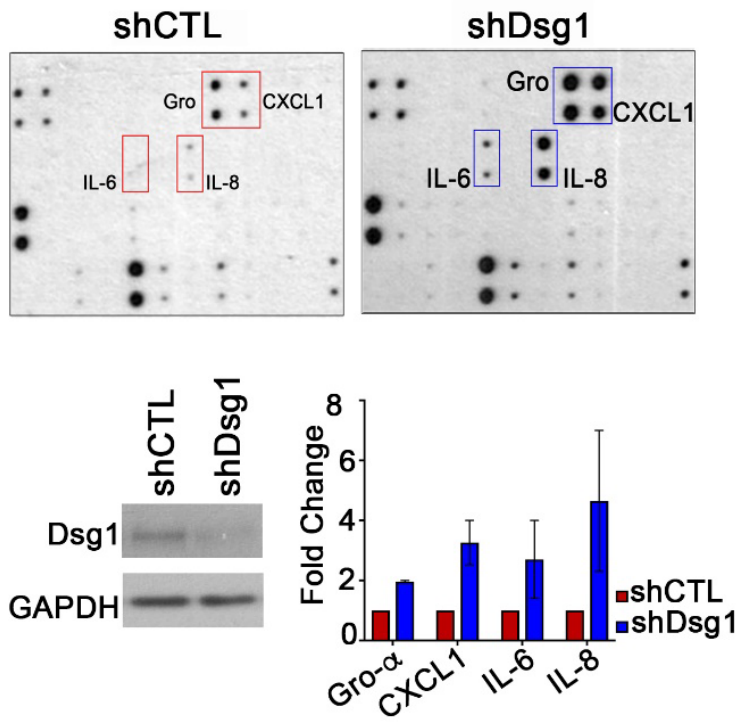
646 Figure 1. Suppression of KC desmoglein 1 increases cytokine production and secretion. (A)

647 NTshRNA (shCTL), Dsg1shRNA (shDsg1), and shDsg1 plus silencing resistant Dsg1-Flag (FL)-

648 infected KCs were induced to differentiate by switching from 0.07 to $1.2 \mathrm{mM} \mathrm{CaCl}_{2}$-containing

649 medium for 72 hours. The mRNA was collected, and cDNA was analyzed for transcription of

650 several cytokines (see Table 1 for additional tested cytokines) ( $\mathrm{N}=3$; * $\left.{ }^{*}<0.03\right)$. (B) Cells with

651 siRNA targeting Dsg3 were also analyzed and found not to have significantly changed levels of

652 cytokines compared to controls $\left(\mathrm{N}=3\right.$; n.s. $=$ not significant, ${ }^{*} \mathrm{p}<0.03$, $\left.{ }^{* * *} \mathrm{p}<0.0002\right)$. (C) Media

653 conditioned from NTshRNA and Dsg1shRNA-infected KCs from days 3-5 after being inducted to

654 differentiate was incubated with a Human Cytokine C3 Array (Raybio) to detect secretion of up to

65542 cytokines, chemokines, and growth factors. Secretion of GRO, CXCL1, IL6, and IL8 were

656 increased upon suppression of Dsg1 compared to controls $(\mathrm{N}=2)$. 
A

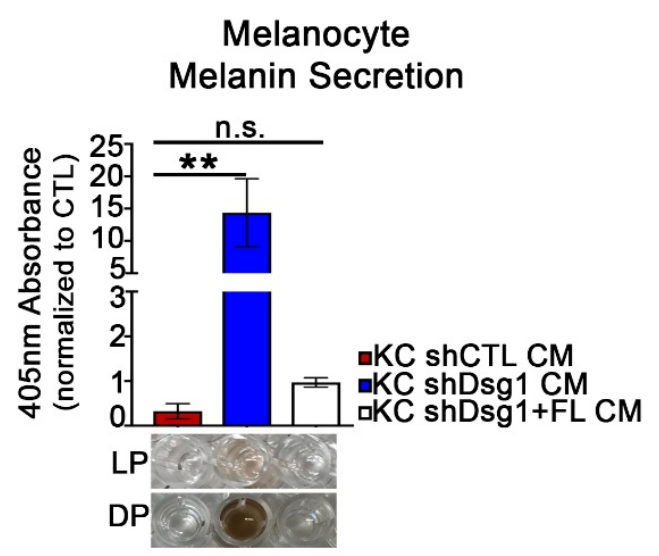

C

Melanocyte

Transcripts

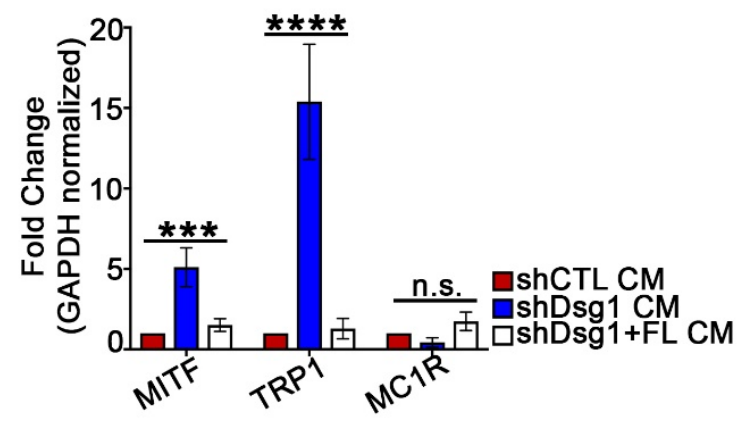

Keratinocyte

Transcripts

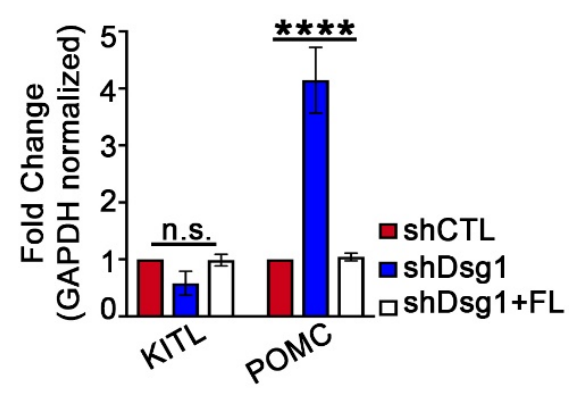

D

Melanocyte Melanin Secretion

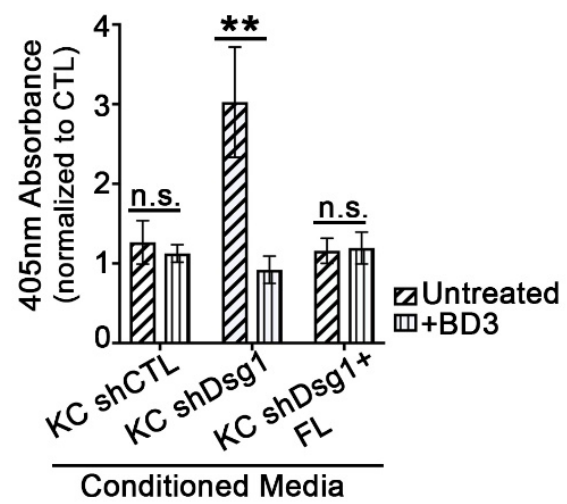

658

659

660

661

662

663

664

665

666

667

Figure 2. Exposure to Dsg1-deficient KC conditioned media increases MC pigment secretion

through classic ligand-dependent mechanisms. (A) Conditioned media (CM) from Dsg1-deficient

(shDsg1) KCs increased MC pigment secretion compared to control (CTL) media or media from KCs expressing exogenous full length Dsg1 (Dsg1FL) (Graph: CM from KC clones were tested on 1 MC clone; n.s. $=$ not significant, $\left.{ }^{* *} \mathrm{p}<0.002\right)$. Multiple clones of MCs were tested with multiple sets of conditioned media (Supplemental Figure 1). Pigment secretion from two MC clones (LP = lightly pigmented, DP = darkly pigmented MC donor) are pictured to indicate the clonal variation but consistent phenomenon. (B) Pro-opiomelanocortin (POMC) mRNA is increased in shDsg1 KCs, while KIT ligand is not changed $\left(\mathrm{N}=3 ;{ }^{* * * *} \mathrm{p}<0.0001\right)$. (C) Transcripts involved in pigment production in MCs (Melanogenesis associated transcription factor [MITF], Tyrosinase-related protein 
668

669

670

671

672

1 [TRP1], and Melanocortin 1 receptor [MC1R]) are upregulated following incubation of MCs with CM from Dsg1 deficient KCs $(\mathrm{N}=3 ; * * * \mathrm{p}<0.0002, * * * * \mathrm{p}<0.0001)$. (D) Inhibiting the MC Melanocortin 1 receptor (MC1R) using recombinant human Beta defensin 3 (BD3) in KC CM inhibits MC pigment secretion $\left(\mathrm{N}=3 ;{ }^{* *} \mathrm{p}<0.002\right)$.
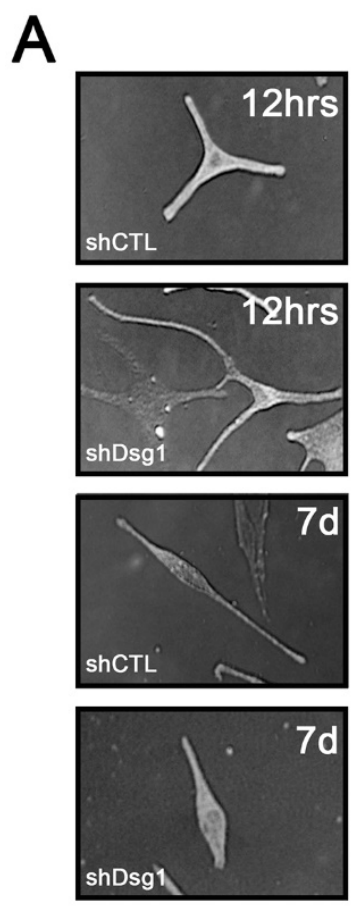
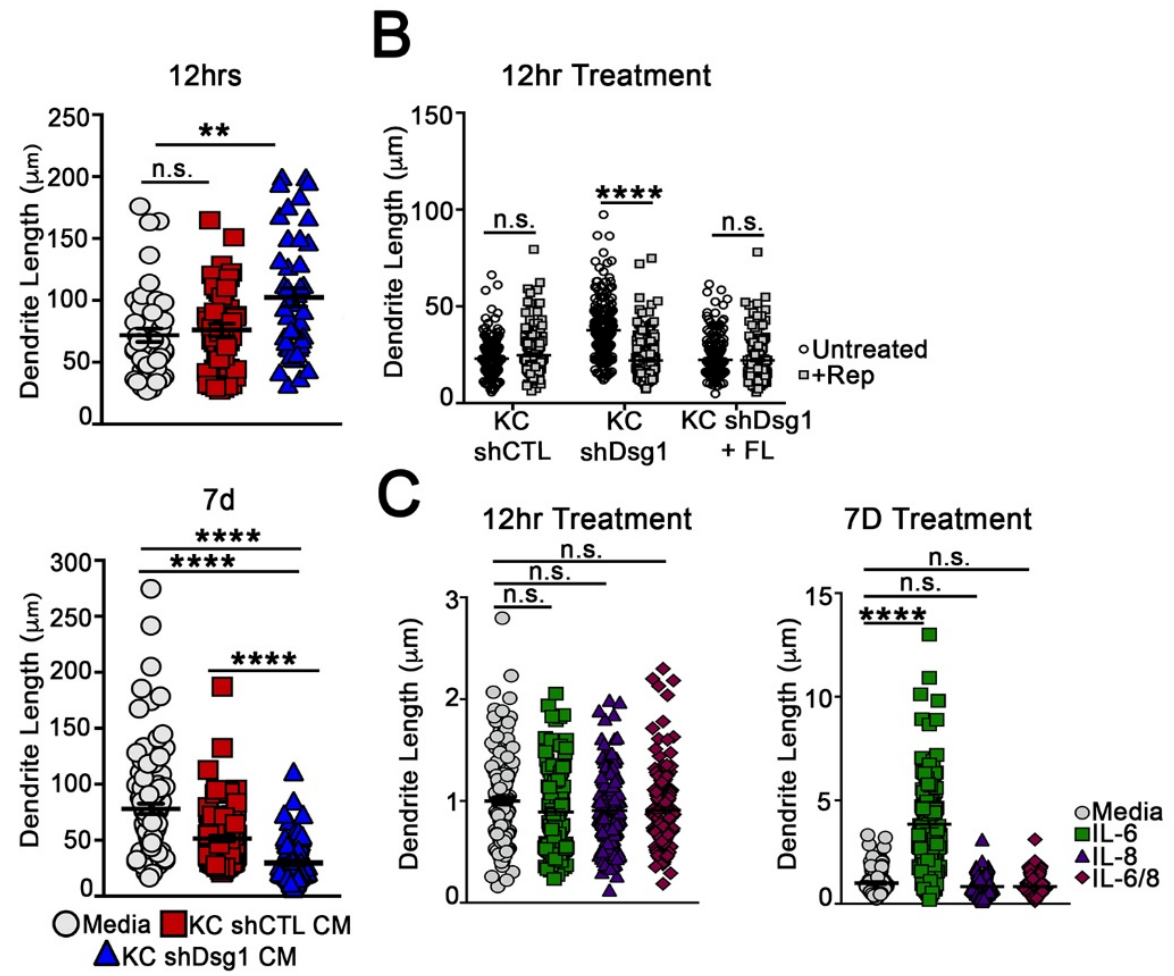

673

Figure 3. Dsg1-deficient KCs change MC dendricity, partially dependent upon

675 cytokine/chemokine signaling. (A) MCs acutely exposed (12 hrs) to NTshRNA or Dsg1shRNA KC

676 conditioned media exhibit increased dendrite length, while long-term exposure (7 days), results in

677 dendrite shortening. ( $\mathrm{N}=3$ experimental replicates, >300 dendrites counted per condition;

$678{ }^{* *} \mathrm{p}<0.002, * * * * \mathrm{p}<0.0001$ ). (B) Incubation of MCs with Reparixin, an inhibitor of the IL8/CXCL1

679 receptor CXCR2, inhibited the increase in MC dendrite length associated with acute exposure (12

680 hours) to Dsg1shRNA KC conditioned media ( $\mathrm{N}=3$ experimental replicates, >150 dendrites counted 
681 per condition; **** $\mathrm{p}<0.0001$ ). (C) Incubation of MCs with IL6, IL8, or IL6 +IL8 in base MC

682 media for 12 hours or 7 days. IL6 significantly increased MC dendrite length but only at the 7 day

683 time point, while IL8 did not have a significant impact on dendrite length ( $\mathrm{N}=3$ experimental

684 replicates, $>150$ dendrites counted per condition; $* * * * \mathrm{p}<0.0001)$.
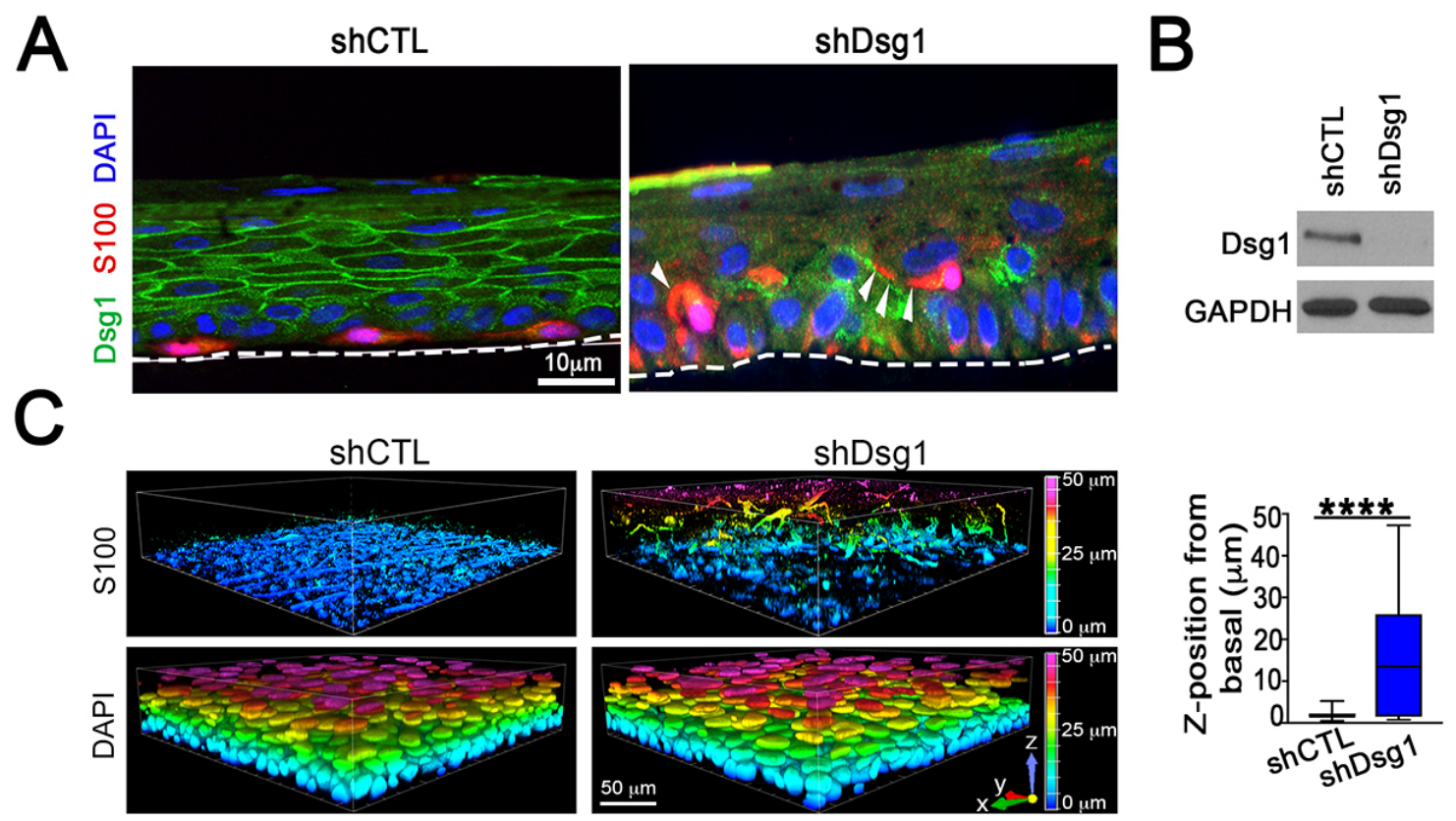

685

Figure 4. MCs are mislocalized within the 3D skin structure when KC Dsg1 is reduced. (A)

687 Silencing Dsg1 in KCs co-cultured with MCs in 3D reconstructed human epidermis results in

688 mislocalization of MCs from basal to suprabasal layers. Dsg1 marks KCs and can be seen to be

689 mostly absent in the shDsg1 culture, S100 marks MCs, DAPI marks nuclei. White arrows indicate

690 MC dendrites. Scale bar $=10 \mu \mathrm{m}$. (B) Immunoblot of Dsg1 protein expression for organotypic

691 cultures pictured in C. GAPDH is a loading control. (C) Images of 3D organotypic cultures of KCs

692 and MCs in presence and absence of Dsg1 prepared using the whole mount method. S100 marks

693 MCs, showing their movement into the suprabasal layers upon Dsg1 reduction ( $N=3$;

$694 * * * * \mathrm{p}<0.0001)$. Horizontal scale bar $=50 \mu \mathrm{m}$, vertical color scale bar $=\mathrm{Z}$ position. Graphical 
A
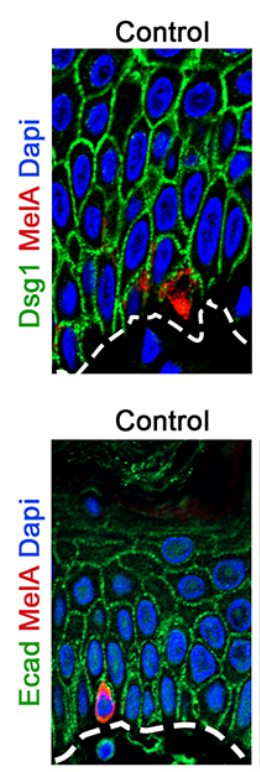



Benign Nevi

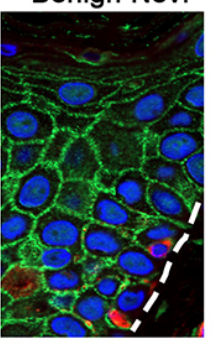

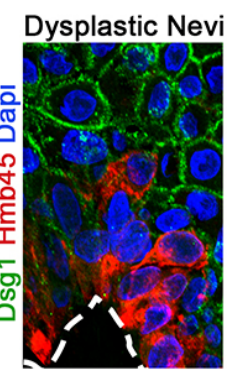

Dysplastic Nevi

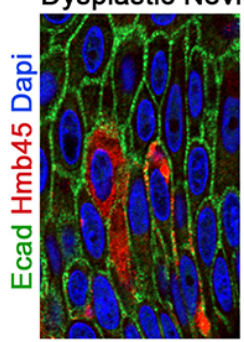

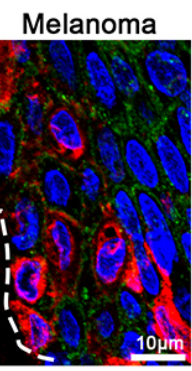

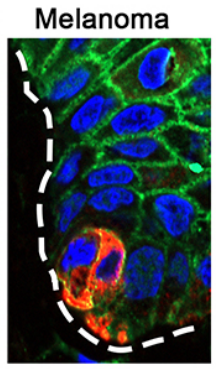

B
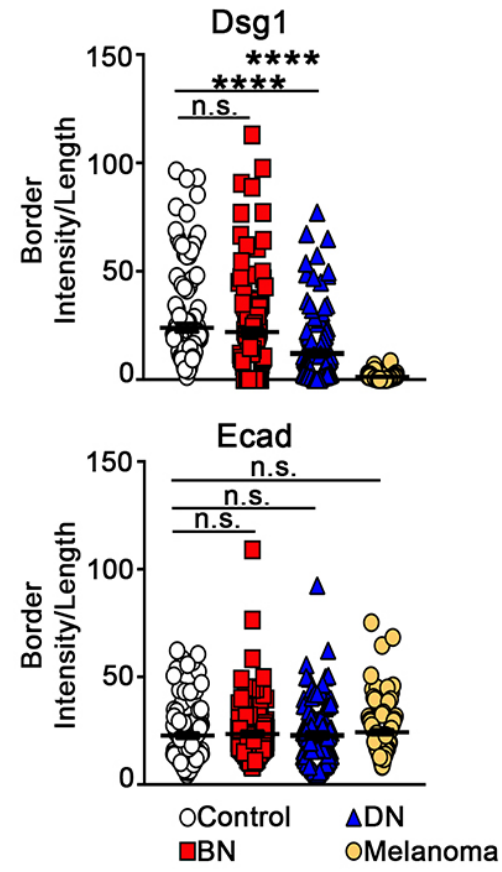

697 Figure 5. Dsg1 levels are significantly reduced at cell-cell borders in the KCs surrounding

698 human dysplastic nevi and melanoma. (A) Staining of Dsg1, Ecad, and the MC markers MelA in

699 control and benign nevi and HMB45 in matched dysplastic and melanoma tissues from the same

700 individuals. (B) Quantification of Dsg1 and Ecad border intensity as compared to control tissue. BN

$701=$ Benign Nevi, DN = Dysplastic Nevi. Scale bar $=10 \mu \mathrm{m} . \mathrm{N}=12 /$ condition (120 borders/condition).

702 $* * * * \mathrm{p}<0.0001$ 


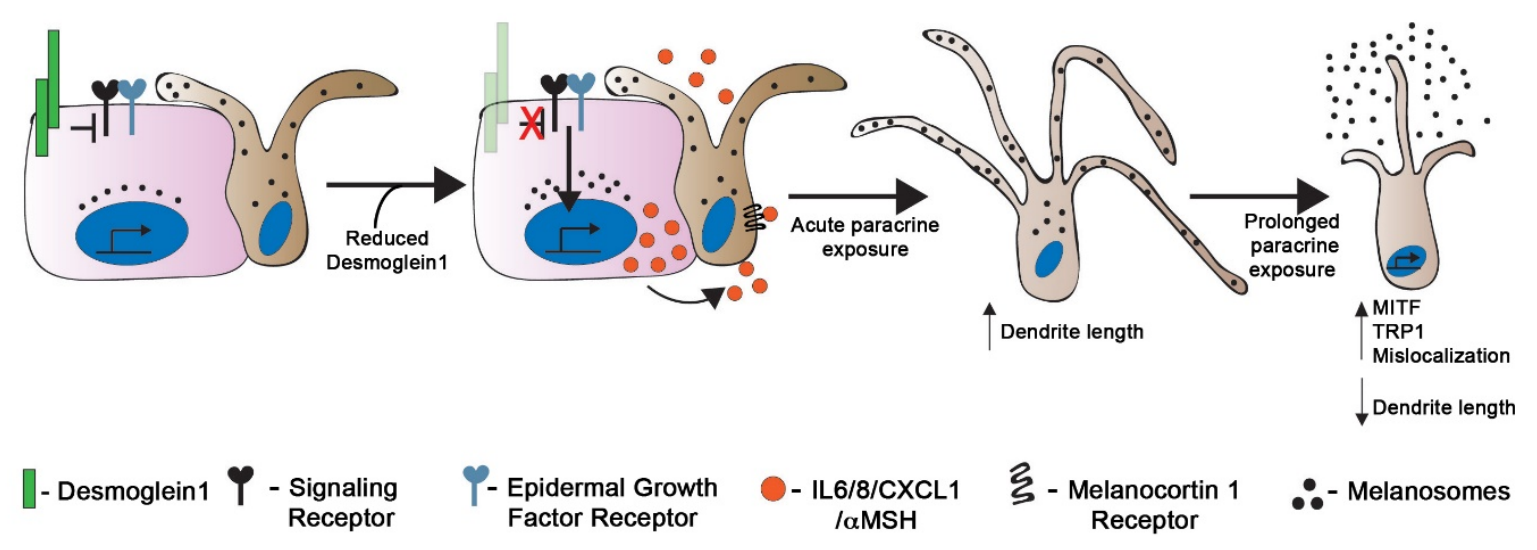

Figure 6. Model: Keratinocyte desmoglein 1 regulates the epidermal microenvironment and

melanocyte behavior. Keratinocytes (pink) and melanocytes (tan) in the skin communicate with each other through both direct contact and paracrine signaling. Reduction in expression of keratinocyte Dsg1 initiates a signaling cascade that results in upregulation and secretion of paracrine factors including IL6, IL8, CXCL1, and $\alpha \mathrm{MSH}$ that affect the signaling, morphology,

710 pigment secretion, and localization of neighboring melanocytes. 


\begin{tabular}{|l|c|c|}
\hline Target & Avg. Fold Change & p-value \\
\hline IL2 & 0.2709 & $* 0.0314$ \\
\hline IL4 & 0.3416 & $* 0.0105$ \\
\hline IL10 & 0.4813 & $* 0.0090$ \\
\hline IL17 & 2555.6718 & 0.1213 \\
\hline IL19 & 58.6191 & 0.1191 \\
\hline IL23 & 40.7489 & 0.0791 \\
\hline CXCL1 & 2.3038 & $* 0.0149$ \\
\hline TNF $\alpha$ & 22.9530 & 0.0816 \\
\hline IFN $\gamma$ & 43.0594 & 0.0686 \\
\hline
\end{tabular}

712 Table 1. Average fold change in KC cytokine transcripts upon depletion of Dsg1 (shCon with

713 shDsg1) to accompany Figure 1A. IL, Interleukin, CXCL1, Chemokine ligand 1; TNF, Tumor

714 Necrosis Factor; IFN, Interferon. Tested by qRT-PCR $(\mathrm{N}=3 * \mathrm{p}<0.03)$.

\begin{tabular}{|l|l|}
\hline Target & \multicolumn{1}{c|}{ Primers } \\
\hline Dsg1 & F: 5'-TCCATAGTTGATCGAGAGGTCAC-3' \\
& R: 5'-CTGCGTCAGTAGCATTGAGTATC-3' \\
\hline Dsg3 & F: 5'-ATCAATGCAACAGATGCAGATGA-3' \\
& R: 5'-TGTCAAAGTGTAGCTGCTGTGT-3' \\
\hline IL1 $\alpha$ & F: 5'-AGTAGCAACCAACGGGAAGG-3' \\
& R: 5'-TGGTTGGTCTTCATCTTGGG-3' \\
\hline IL1 $\beta$ & F: 5'-GCAAGGGCTTCAGGCAGGCCGCG-3’ \\
\hline
\end{tabular}




\begin{tabular}{|c|c|}
\hline & R: 5’-GGTCATTCTCCTGGAAGGTCTGTG-3’ \\
\hline IL2 & $\begin{array}{l}\text { F: 5’-GTCACAAACAGTGCACCTAC-3’ } \\
\text { R: 5'-CCCTGGGTCTTAAGTGAAAG-3' }\end{array}$ \\
\hline IL4 & $\begin{array}{l}\text { F: 5’-ACTTTGAACAGCCTCACAGAG-3’ } \\
\text { R: 5’-TTGGAGGCAGCAAAGATGTC-3’ }\end{array}$ \\
\hline IL6 & $\begin{array}{l}\text { F: 5’- ACAGCCACTCACCTCTTCAG -3’' } \\
\text { R: 5’- CCATCTTTTTCAGCCATCTTT -3’ }\end{array}$ \\
\hline IL8 & $\begin{array}{l}\text { F: 5’- ATGACTTCCAAGCTGGCCGT -3’ } \\
\text { R: 5’- TCCTTGGCAAAACTGCACCT -3’' }\end{array}$ \\
\hline IL10 & $\begin{array}{l}\text { F: 5’- GGTTGCCAAGCCTTGTCTGA -3’ } \\
\text { R: 5’- AGGGAGTTCACATGCGCCT -3’ }\end{array}$ \\
\hline IL17 & $\begin{array}{l}\text { F: 5’- TCAACCCGATTGTCCACCAT-3’ } \\
\text { R: 5’- GAGTTTAGTCCGAAATGAGGCTG -3’ }\end{array}$ \\
\hline IL19 & $\begin{array}{l}\text { F: 5’- GAGCCATCCAAGCTAAGGACA -3’ } \\
\text { R: 5’- CTTGGTCACGCAGCACACAT -3’ }\end{array}$ \\
\hline IL23 & $\begin{array}{l}\text { F: 5’- GCTTCAAAATCCTTCGCAG -3’ } \\
\text { R: 5’- TATCTGAGTGCCATCCTTGAG -3' }\end{array}$ \\
\hline CXCL1 & $\begin{array}{l}\text { F: 5’ AACCGAAGTCATAGCCACAC -3' } \\
\text { R: 5’- GTTGGATTTGTCACTGTTCAGC -3' }\end{array}$ \\
\hline $\mathrm{TNF} \alpha$ & $\begin{array}{l}\text { F: 5’ ATGAGCACTGAAAGCATGATCC -3’' } \\
\text { R: 5’- GAGGGCTGATTAGAGAGAGGTC -3’' }\end{array}$ \\
\hline IFN $\gamma$ & $\begin{array}{l}\text { F: 5’- CTAATTATTCGGTAACTGACTTGA -3’ } \\
\text { R: 5'- ACAGTTCAGCCATCACTTGGA -3’ }\end{array}$ \\
\hline
\end{tabular}




\begin{tabular}{|c|c|}
\hline POMC & $\begin{array}{l}\text { F: 5’- AGGCACTTGCTGGATTCTCC -3’’ } \\
\text { R: 5’- GCCCTTCTTGTAGGCGTTCT -3’' }\end{array}$ \\
\hline MITF & $\begin{array}{l}\text { F: 5’- TTATAGTACCTTCTCTTTGCCAGTCC -3’ } \\
\text { R: 5’- CTTATAAAATCССТCTTTTTCACAGTTGGA -3’’ }\end{array}$ \\
\hline MC1R & $\begin{array}{l}\text { F: 5’- ACTTCTCACCAGCAGTCGTG -3’' } \\
\text { R: 5’- CATTGGAGCAGACGGAGTGT -3’ }\end{array}$ \\
\hline TRP1 & $\begin{array}{l}\text { F: 5’- GTGCCACTGTTGAGGCTTTG -3’' } \\
\text { R: 5’- ATGGGGATACTGAGGGCTGT -3’ }\end{array}$ \\
\hline KITL & $\begin{array}{l}\text { F: 5’- TCGATGACCTTGTGGAGTGC -3’' } \\
\text { R: 5’- TGCTGTCATTCCTAAGGGAGC -3’ }\end{array}$ \\
\hline ET1 & $\begin{array}{l}\text { F: 5'- GACATCATTTGGGTCAACACTC } \\
\text { R: 5'- GGCATCTATTTTCACGGTCTGT }\end{array}$ \\
\hline GAPDH & $\begin{array}{l}\text { F: 5'- ACCACAGTCCATGCCATCAC -3' } \\
\text { R: 5'- TCCACCACCCTGTTGCTGTA -3' }\end{array}$ \\
\hline
\end{tabular}

717 Table 2. Primers used in this study. Dsg1, Desmoglein 1; Dsg3, Desmoglein 3; IL, Interleukin,

718 CXCL1, Chemokine ligand 1; TNF, Tumor Necrosis Factor; IFN, Interferon; POMC, Pro-

719 opiomelanocortin; MITF, Melanogenesis associated transcription factor; MC1R, Melanocortin 1

720 receptor; TRP1, Tyrosinase related protein 1; KITL, Kit ligand; ET1, Endothelin 1; GAPDH,

721 Glyceraldehyde 3-phosphate dehydrogenase. 